\title{
神经反射在一足致炎大鼠对侧非致炎足 伤害感受性变化中的作用
}

\author{
罗蕾吕国蔚
}

(首都医学院神经生物学研究室, 北京 100054)

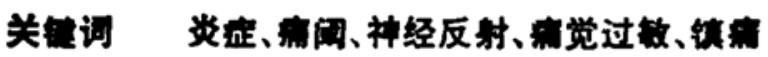

我们先前的工作表明, 一足致炎大鼠对侧非致炎足的痛阈出现以升高变化为主的显著降 低、不变和显著升高等 3 种变化; 这 3 种变化分别与致炎足痛阈降低的重、中、轻显著相关 ${ }^{\omega}$. 本工作旨在进一步观察与分析神经因素在非致炎足伤害感受性变化中的作用.

\section{一、材料与方法}

㫿性 Wistar 大鼠 56 只,体重 180 - 280g。按随机原则, 将动物分为致炎足同侧神经切 断组与相应假处理组.

按文献 [1-3] 测定各组各鼠双足的基础痛阈后, 以 $4 \%$ 戊巴比妥钠腹腔麻醉 $(40 \mathrm{mg} / \mathrm{kg})$ 行无菌手术,随机暴露上述两组动物一侧的坐骨神经和股神经.

术后 3 天, 再测各组各鼠双足术后基础痛阈后, 向神经暴笿侧足蹠注射 $0.1 \%$ 鹿角菜 0.1 $\mathrm{ml}$, 测定各组各鼠注射后 30 及 $60 \mathrm{~min}$ 时双足的痛闻. 然后, 向实验组动物坐骨神经干内注 射 $2 \%$ 普鲁卡因 $0.01 \mathrm{ml}$, 并以漫有该局部麻药的细棉芯缠绕于股神经上, 向假处理组相应的 神经施加等量的生理盐水. $1 \mathrm{~min}$ 后, 切断实验组的坐骨神经及股神经. 继续测定鹿角菜注 射后 $120 、 150$ 和 $180 \mathrm{~min}$ 时各组各鼠非致炎足的痛阈。

所测定的数据输人计算机，进行方差分析（ANOVA）和两两分析，显著性水平为 0.05 .

\section{二、实验结果}

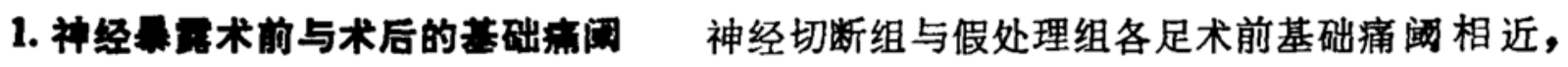
均在 10132.472-10799.082Pa 范围, 术后3天各鼠各足的基础痛间分布在 10532.438 $11065.726 \mathrm{~Pa}$ ，同一组动物左、右足之间及术前与术后基础痛阈之间均未见显著差异.

2. 致炎制注射后致炎足与非致炎足家问的变化 一足致炎后, 实验组与假处理组致炎 足的痛闻显著下降 (表 $1 、 2$, 图 1、2). 对侧非致炎足的痛间出现显著降低、不变和显著开高 等 3 个方向的变化. 实验组 (34 例) 中 12 例非致炎足痛阈呈降低变化, 致炎剂注射后 30 及 $60 \mathrm{~min}$ 时的痛闻与 $0 \mathrm{~min}$ 时的痛闻之间有显著或非常显著的差异; 6 例痛阈保持不变; 16 例

1991-06-28收稿, 1992-04-01 收售改稿 


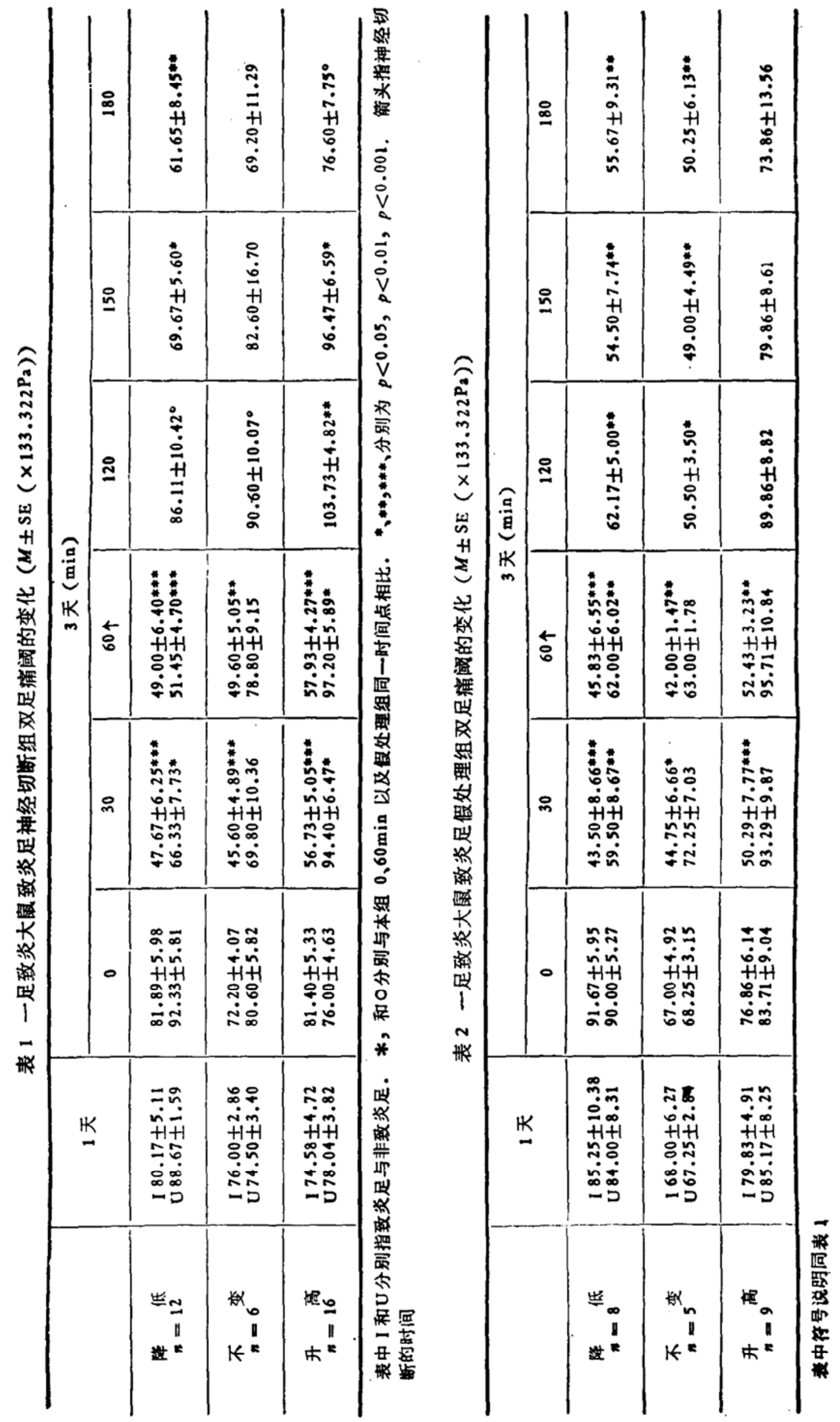


星升高变化,与 $0 \mathrm{~min}$ 相比有显著差异(表 1 ,图 1).

假处理组非致炎足的痛阘亦出现与实验组相似的降低、不变和开高等变化(表 2、国 2)。
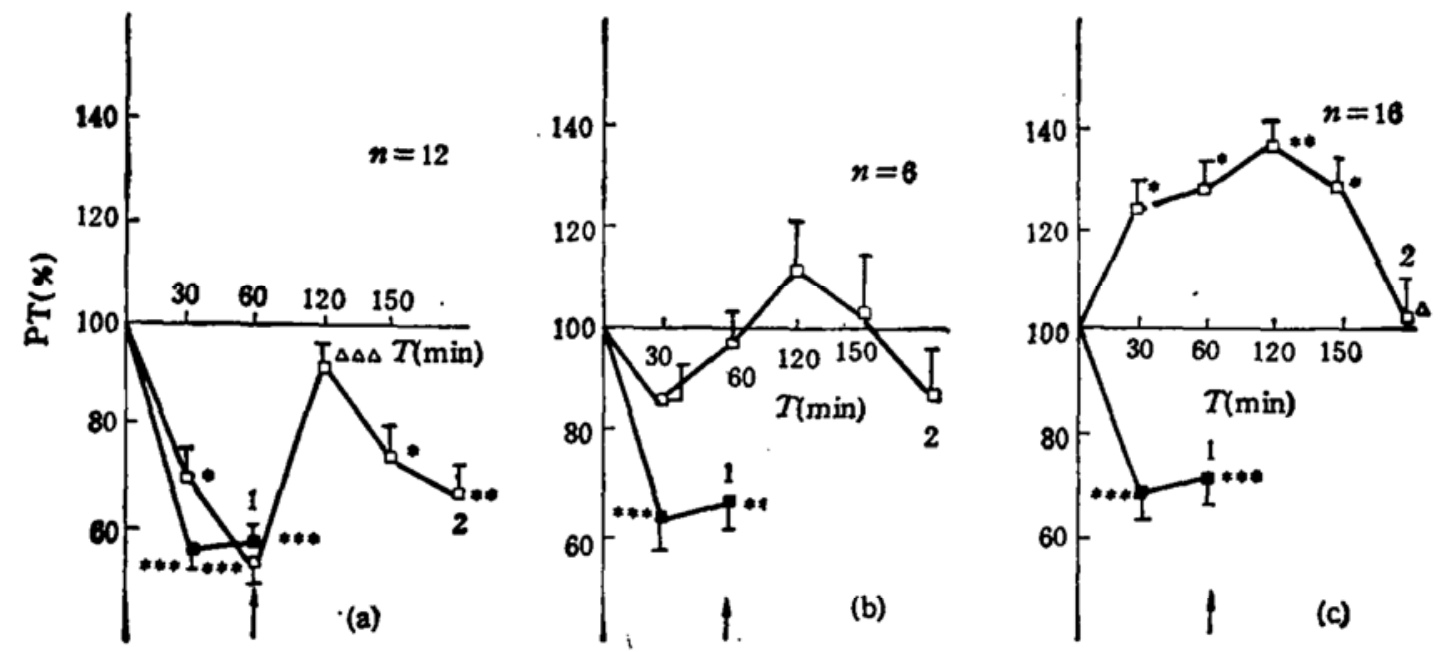

图 1 致炎剂注射侧神经切断组双足痛阈的变化

(a)、(b)、(c) 分别表示致炎后对侧非致炎足痛間发生降低、不变和升高的动物双足痛阘的变化. 纵坐 标: 以 $0 \mathrm{~min}$ 时的新为 $100 \%$ 的痛闻变化的百分比, 横坐标: 致炎后 $(0 \mathrm{~min}) 30 、 60 、 120 、 150$ 和 $180 \mathrm{~min}$ 时的洞痛时间. 1 - 致炎足; 2 - 非致炎足. $* p<0.05, * p<0.01, * * p<0.001$. $\uparrow$ 指神经切断的时问
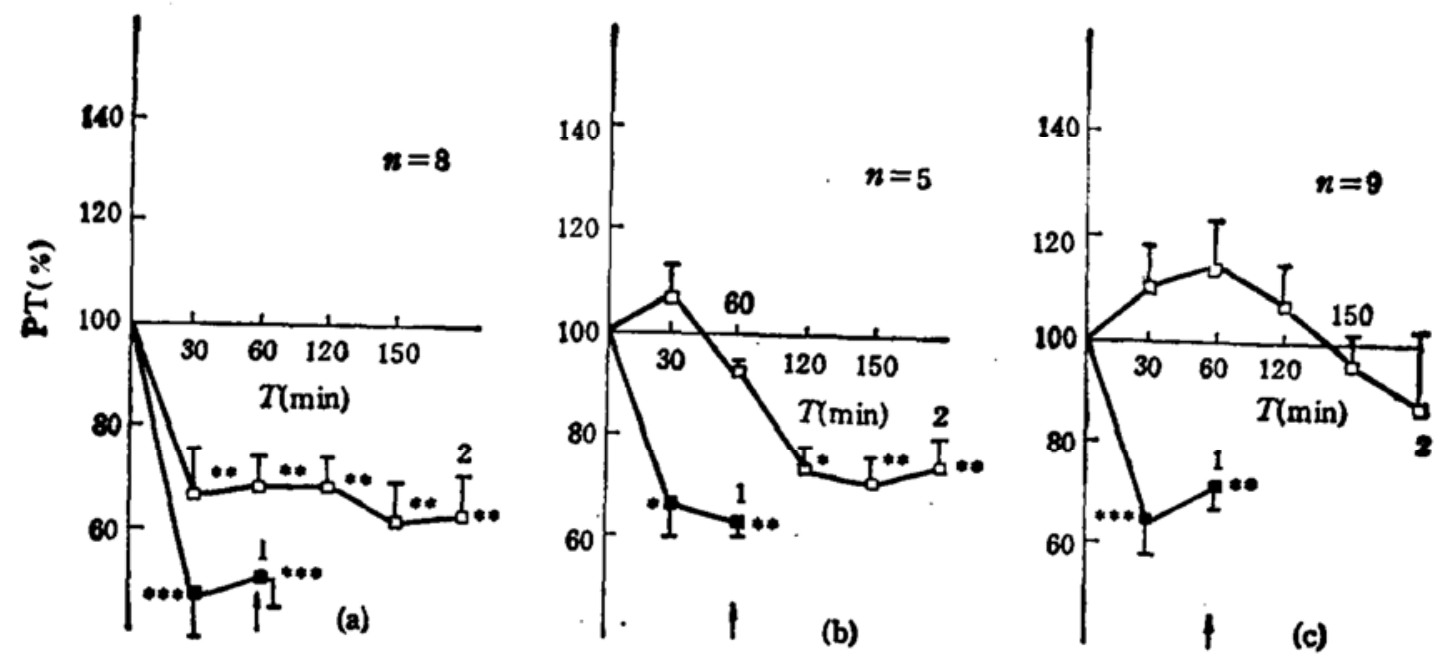

图 2 致炎剂注射侧假处理组双足痛阈的变化

图内说明同图 1

统计处理表明,实验组非致炎足痛闻发生降低、不变和升高变化的动物, 其术前与术后的 基础痛阘, 以及注射后 30 及 $60 \mathrm{~min}$ 的痛阈与假处理组相应动物上述各时间点相比, 均未见 显著差异 (表 1, 表 2)。

3. 坐神经及股神经切断后非数炎足来问的变化 神经切断后, 痛闻原呈降低变化者 转呈升高的变化,在 $120 \mathrm{~min}$ 时, 由神经切断前 $(60 \mathrm{~min})$ 的 6871.4158 升高至 $11480.357 \mathrm{~Pa}$, 提高了 $37.54 \%$, 差异非常显著(表 1, 图 1(a)), 痛阈原星不变或升高的动物, $120 \mathrm{~min}$ 时的 
痛酒虽较各该组神经切断前略有升高, 但统计处理未见显著差异(表 1, 图 1(b)、(c)).

假处理组痛闻发生降低、不变及升高变化的动物,在假处理后, 痛阀略有降低,但各时间点 痛阀与处理前相比,未见显著差异(表 2,图 2(a)、(b)、(c)).

统计处理表明,神经切断后, 痛阈原呈降低及不变的动物, 与相应假处理组在 $120 \mathrm{~min}$ 时 的痛阀相比有显著差异(实验组不变组: 假处理组不变组; 实验组降低组: 假处理组降低组. $120 \mathrm{~min}, P<0.05)$; 实验组呈升高变化者, 在神经切断后各时间点的痛闻与假处理组相应时 间点相比,未见显著差异(表 $1 、 2$, 图 1、2).

\section{三、讨 论}

1985 年, Levine 等提出神经反射性炎症的观点 ${ }^{[1}$, 认为局部组织损伤或发炎时, 其对侧 或远隔部位的伤害感受性变化是通过神经反射引起的痛觉过敏,而不存在体液因素的影响.但 是, Levine 的说法不能全面解释我们先前工作和本工作所报道的一足致炎后, 对侧非致炎 足除产生痛敏外,还产生痛觉正常及镇痛等两个方向的变化.

Levine 等利用竦椒素预先损毁无骴传人纤维，用吸乙啶造成节后交感神经传出纤维 变,并用急、慢性神经切除等方法, 均证明上述神经因素可以引起非致炎足的痛敏及促进肿胀

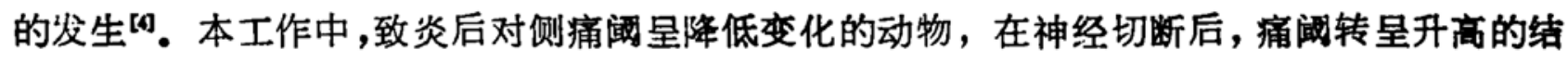
果与 Levine 的结果一致,提示神经反射参与对侧足痛阈降低的过程. 但是, Levine 等的说 法不能解释本工作中痛间呈升高变化及痛诚不变的两组动物, 致炎侧神经切断后, 对侧非致炎 足的痛菏未出现显著变化的结果.

我们在文献 [1] 中曾提出,局部组织损伤后,在产生神经反射性炎症的同时，机体还产生 抗痛过程;致炎后,致痛因索可以通过神经及/或体液途径影响致炎侧和对侧健足,使之产生痛 敏; 同时,损伤或致炎局部也可产生某种抗痛物质,抵制致痛因素的作用,并经体液途径扩散至 对侧健部和其他远隔部位,引起镇府效应; 致炎较轻时, 致痛因素的浓度较低, 而抗痛物质的浓 度相对较高, 抗痛物质则可有效地抑制致痛物质的作用. 致炎较重者, 致痛因素的浓度高于抗 痛物质时, 经神经反射或体液扩散引起痛敏; 致炎程度居中时, 致痛因素与抗痛物质双方力均等, 则不引起痛觉的明显变化. 本工作的结果支持这一推论.

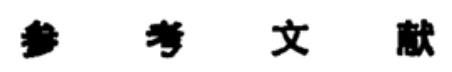

[1]吕国蒋、罗者、店肪,生理学报，43(1991),1: 78-83.

[2] 孙志强、吕国薜、店昉,生理学报, 40(1988), 608-613.

[3] 店昉、吕国莭、罗暮, 首都医学院学报, 10(1989),3: 229--231.

[4] Levine, J.D., Dardick, S.J., Basbaum, A.I. et al., J. Newrosci., 5 (1985), 5:1380-1386. 